\title{
PCR-Introduced Loop Structure as Primer in DNA Sequencing
}

BioTechniques 25:876-884 (November 1998)

\section{Mostafa Ronaghi, Bertil Pettersson, Mathias Uhlén and Pål Nyrén}

The Royal Institute of Technology, Stockholm, Sweden

\begin{abstract}
The need for a primer hybridization step before sequencing has been eliminated using a stem-loop structure generated by $P C R$. The loop structure is obtained by careful design of the PCR primer or by cloning the target DNA into a dedicated vector (pRIT 28HP). After solid-phase capture of the PCR product, the loop is formed by elution of the non-bound strand. Here, we show that both the immobilized and the eluted strand can be analyzed using conventional Sanger DNA sequencing and the novel pyrosequencing method as described previously. By using a stem-loop structure as a primer for DNA sequencing, the risk for mispriming is minimized.
\end{abstract}

\section{INTRODUCTION}

Over the past years, the polymerase chain reaction (PCR) has widely been used to produce DNA template for direct sequencing of genomic or cloned material. Modifications of this technique have improved the strategies for DNA sequencing, most notably, boomerang DNA amplification (4), fluorescent Sanger DNA sequencing $(13,17)$, solid-phase DNA sequencing (5), cycle sequencing (7) and cDNA sequencing (1). Thus far, developments have focused on the original Sanger DNA sequencing technique (12). Recently, Ronaghi et al. described a bioluminometric method for solid-phase DNA sequencing (9). This sequencing-by-synthesis method was later improved by the introduction of a nucleotide-degrading enzyme into the reaction mixture, thus eliminating the need for a washing step between each nucleotide addition (10). However, in all sequencing-bysynthesis methods, there is a risk for primer loss and possible repriming, which could result in a decreased signal-to-noise ratio. In addition, false signals can also be generated by mispriming. In this study, we show that these problems can be reduced by introducing a covalently attached primer by PCR. We also show that the efficiency of the DNA polymerization reaction is strongly influenced by the stem length.

\section{MATERIALS AND METHODS}

\section{Synthesis and Purification of Oligonucleotides}

Oligonucleotides were synthesized by phosphoramidite chemistry and purified with HPLC (Interactiva, Ulm, Germany) (see Table 1).

\author{
Construction of the Hairpin Vector \\ pRIT 28HP and Template Prepara- \\ tion
}

The oligonucleotides RIT 203 and RIT 204 were hybridized and ligated to HindIII (Amersham Pharmacia Biotech, Uppsala, Sweden) pre-restricted plasmid pRIT 28 (6) (the obtained plasmid was named pRIT 28HP). PCRs were performed on the multilinker of plasmid pRIT 28HP with $7.5 \mathrm{pmol}$ of the following primer pairs: RIT 29/ ROMO 205S, RIT 29/ROMO 205L, RIT 29/ROMO 206, RIT 29/ROMO 207, RIT 29/ROMO 208 or RIT 27/ROMO 205B. The reactions were performed in $0.2 \mathrm{mM}$ dNTP, $1 \mathrm{U}$ of AmpliTaq ${ }^{\circledR}$ DNA Polymerase (PE Applied Biosystems, Foster City, CA, USA) or $P f u$ DNA Polymerase (Stratagene, La Jolla, CA, USA) and Pfu reaction buffer (Stratagene) for the enzymes, making up a final volume of 50 $\mu \mathrm{L}$. The temperature profile included a 15 -s denaturation step at $95^{\circ} \mathrm{C}$ and a 90 s hybridization/extension step at $72^{\circ} \mathrm{C}$. These steps were repeated 30 times with a GeneAmp ${ }^{\circledR}$ PCR System 9600 (PE Applied Biosystems). The PCR products were immobilized onto streptavidin-coated super paramagnetic Dynabeads ${ }^{\circledR}$ according to the supplier (Dynal AS, Oslo, Norway). Singlestranded (ss)DNA templates, either immobilized (obtained from the RIT 29/ROMO 205S, RIT 29/ROMO 205L, RIT 29/ROMO 206 or RIT 27/ROMO 205B amplified reactions) or non-immobilized (from the RIT 27/ROMO 205B amplified reaction), were obtained by alkali treatment and used for DNA sequencing as described below.

\section{Conventional DNA Sequencing on Stem-Loop Structure}

Solid-phase Sanger DNA sequenc- 
ing was carried out according to Sambrook et al. (11) using [ $\left.{ }^{35} \mathrm{~S}\right] \mathrm{dATP}$ labeling with Klenow Fragment DNA Polymerase (Amersham Pharmacia Biotech) at either $25^{\circ}$ or $37^{\circ} \mathrm{C}$. The produced Sanger fragments were released from the loop-structures by restriction with $B g$ III restriction endonuclease (Amersham Pharmacia Biotech) followed by addition of formamide and heat treatment before loading on a $6 \%$ polyacrylamide gel. The gel was analyzed by applying a photo-film overnight to obtain an autoradiogram.

\section{DNA Sequencing by Pyrosequencing} on Stem-Loop Structures

The PCR-generated loop structures, immobilized for solid-phase pyrosequencing (9) and either immobilized or non-immobilized for liquid-phase pyrosequencing (10), were incubated with either Sequenase ${ }^{\circledR}$ Version 2.0 or exo $^{-}$ Klenow DNA Polymerase (Amersham Pharmacia Biotech). The sequencing procedure was carried out as described earlier $(9,10)$.

\section{Influence of the Stem-Length on Polymerization Efficiency}

Stem-loop structures with different stem-length, ranged from 6-18-bp long, were incubated with either Sequenase Version 2.0 or exo- Klenow DNA polymerase. The polymerization reactions were studied by addition of 100 pmol deoxynucleotide and the rates were determined by the enzymatic luminometric inorganic pyrophosphate detection assay (ELIDA) (8).

\section{RESULTS}

\section{Generation of a Dedicated Plasmid pRIT 28HP}

A novel plasmid, based on pRIT 28 (6), was constructed (see Materials and Methods) containing a hairpin-like stem-loop structure downstream of the multi-cloning site (Figure 1). This allows the use of general PCR primers to amplify any insert introduced into the multi-cloning site. Using a biotinylated primer, the PCR product can be immobilized onto streptavidin-coated paramagnetic beads. Thus, ssDNA template
Table 1. Oligonucleotide Sequences

\begin{tabular}{|lc|}
\hline Oligonucleotide & Sequence \\
\hline RIT 29 & See Reference 5 \\
RIT 27 & See Reference 5 \\
RIT 203 & 51-mer: 5'-AGCTTGGGTTCGAGGAGATCTTCCGGGTTA- \\
& CGGCGGAAGATCTCCTCGAGG-3' \\
ROMO 208 & 16-mer: 5'-CTTCCGGGTTACGGCG-3' \\
ROMO 207 & 19-mer: 5'-GATCTTCCGGGTTACGGCG-3' \\
RIT 204 & 51-mer: 5'-AGCTCCTCGAGGAGATCTTCCGCCGTAACC- \\
& CGGAAGATCTCCTCGAACCCA-3' \\
ROMO 205S & 25-mer: 5'-CGAGGAGATCTTCCGGGTTACGGCG-3' \\
ROMO 205B & 25-mer: 5'-biotin-CGAGGAGATCTTCCGGGTTACGGCG-3' \\
ROMO 205L & 28-mer: 5'-CCTCGAGGAGATCTTCCGGGTTACGGCG-3' \\
ROMO 206 & 22-mer: 5'-GGAGATCTTCCGGGTTACGGCG-3' \\
& \\
\hline
\end{tabular}

can be obtained by alkali treatment as described earlier (5). The ssDNA will spontaneously form a 13-bp-long stem after a loop of 8 bases and a free $3^{\prime}$ end suitable for primer extension. A $B g l I I$ restriction site was engineered into the stem part of the structure to allow reloop structure after the Sanger sequencing reaction. Figure 2 shows the results striction and subsequent removal of the

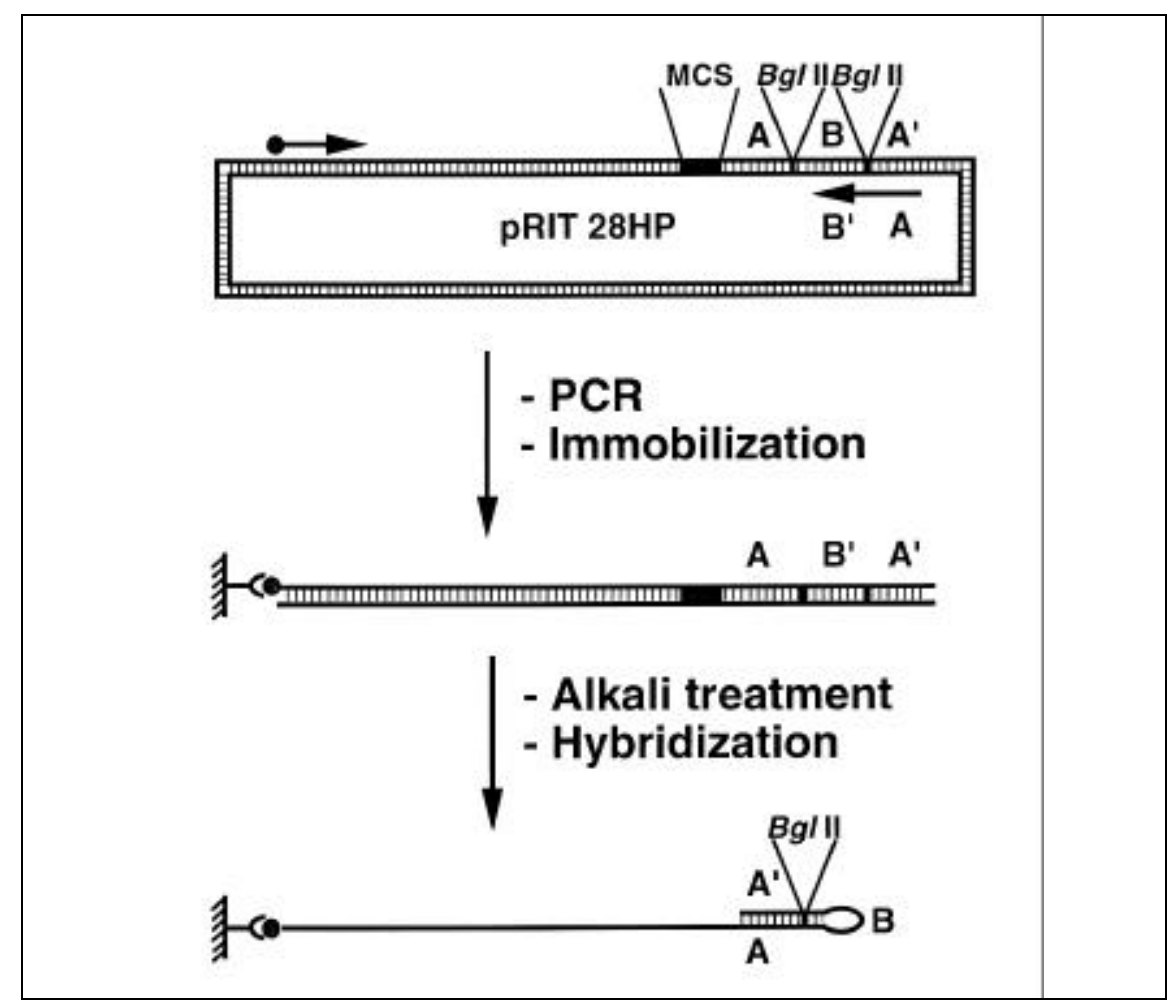

Figure 1. A schematic representation of stem-loop generation using pRIT 28HP. A primer is hybridized to a cassette containing a stem-loop structure downstream of the multi-cloning site. After amplification, the PCR product is immobilized on a solid-support, and a single-stranded template with a covalently attached primer is obtained after alkali treatment. The generated fragment with free $3^{\prime}$ end could be used for DNA sequencing. $\mathrm{A}^{\prime}$ at the primer indicates template-specific sequence, $\mathrm{B}^{\prime}$ is the loop-forming region and $\mathrm{A}$ and $\mathrm{B}$ are the reverse complementary parts of $\mathrm{A}^{\prime}$ and $\mathrm{B}^{\prime}$, respectively. BglII and MCS indicate restriction sites for $B g l I I$ endonuclease and multi-cloning sites, respectively. The arrows indicate primer sites and the direction of synthesis. The blob indicates the biotin moiety. 
from Sanger DNA sequencing using radiolabeled dATP. The Sanger fragments were released from the magnetic beads by restriction before loading on the electrophoresis gel. The results show that good quality data are obtained at two different temperatures $\left(25^{\circ}\right.$ and $37^{\circ} \mathrm{C}$ ) with or without hybridization step.

\section{Introduction of Stem-Loop Structure by Primer Design}

The use of dedicated vectors to obtain the stem-loop structure has several disadvantages, such as the need to clone the target DNA into a special vec-

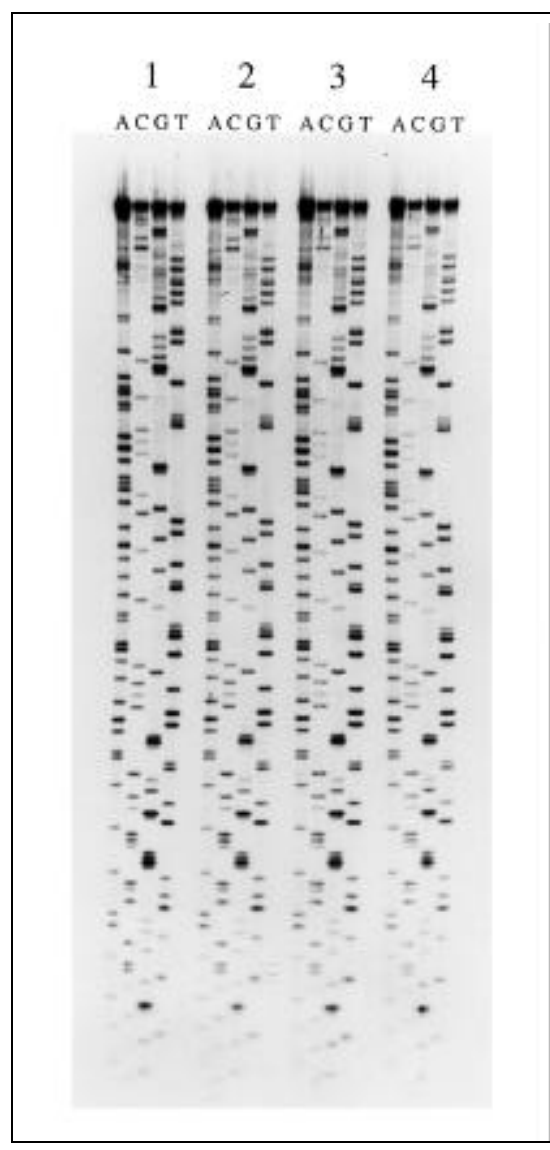

Figure 2. Autoradiograph obtained from Sanger DNA sequencing, performed on a 161base-long self-primed PCR product. The Sanger fragments were released after restriction with $B g l$ II endonuclease. Lane 1: reaction with Klenow polymerase performed at $25^{\circ} \mathrm{C}$ with an additional hybridization step. Lane 2 : reaction with Klenow polymerase performed at $25^{\circ} \mathrm{C}$ without an additional hybridization step. Lane 3: reaction with Klenow polymerase performed at $37^{\circ} \mathrm{C}$ with an additional hybridization step. Lane 4: reaction with Klenow polymerase performed at $37^{\circ} \mathrm{C}$ without an additional hybridization step. tor before sequencing. To allow direct sequencing on any target DNA, an alternative strategy was developed. The strategy relies on the design of a $5^{\prime}$ "handle" on the PCR primer that is the reverse complement to the 3 ' end of the primer (Figure 3). After immobilization of the PCR product using a biotinylated primer, ssDNA with an intrinsic sequencing primer is obtained by alkali treatment. By introduction of biotin to either the handle primer or the other primer, the stem-loop structure is obtained either on the immobilized or the eluted soluble strand (Figure 3 ). To circumvent restriction cleavage and gel electrophoresis necessary for conventional sequencing, the recently developed methods for pyrosequencing were used $(9,10)$. These methods are based on the iterative addition of four different nucleotides into a mixture of three (9) or four (10) enzymes. Release of pyrophosphate (PPi) as a result of nucleotide incorporation is detected by ELIDA (8). Unreacted nucleotides can be removed either by washing (9) or by enzymatic degradation (10).

\section{The Effect of the Stem-Length after the Loop on the Polymerization Efficiency}

The solid-phase pyrosequencing approach (9) was used to study the effect of the stem-length after the loop on the polymerization efficiency. Different stem-loop structures were extended with either Sequenase Version 2.0 or exo- Klenow DNA polymerase. The initial rate of polymerization was followed by ELIDA. The traces shown in Figure $4 \mathrm{a}$ demonstrate that neither Klenow nor Sequenase are showing catalytic activity on a 6-bp-long stem after the loop. Only Klenow showed catalytic activity on a 9-bp-long stem (Figure 4b). However, the rate was low and full extension was obtained after $2.5 \mathrm{~min}$. On the 10-bp-long stem, Klenow showed much faster polymerization rate (data not shown), while Sequenase was still unable to incorporate nucleotides. On the 12-bp-long stem, Klenow showed rapid polymerization rate, while Sequenase showed slow polymerization rate (Figure $4 c$ ). On the 15-bp-long stem, both exo- Klenow and Sequenase were able to extend the primer at a high rate (Figure 4d). Similar results were obtained with the 18bp-long stem for both polymerases (Figure 4e).

\section{Pyrosequencing}

Solid-phase pyrosequencing (Figure 5) and liquid-phase pyrosequencing (Figure 6) were performed using exoKlenow DNA polymerase on a template having a stem of $15 \mathrm{bp}$. Identical results were obtained when using Sequenase (data not shown). Note that the

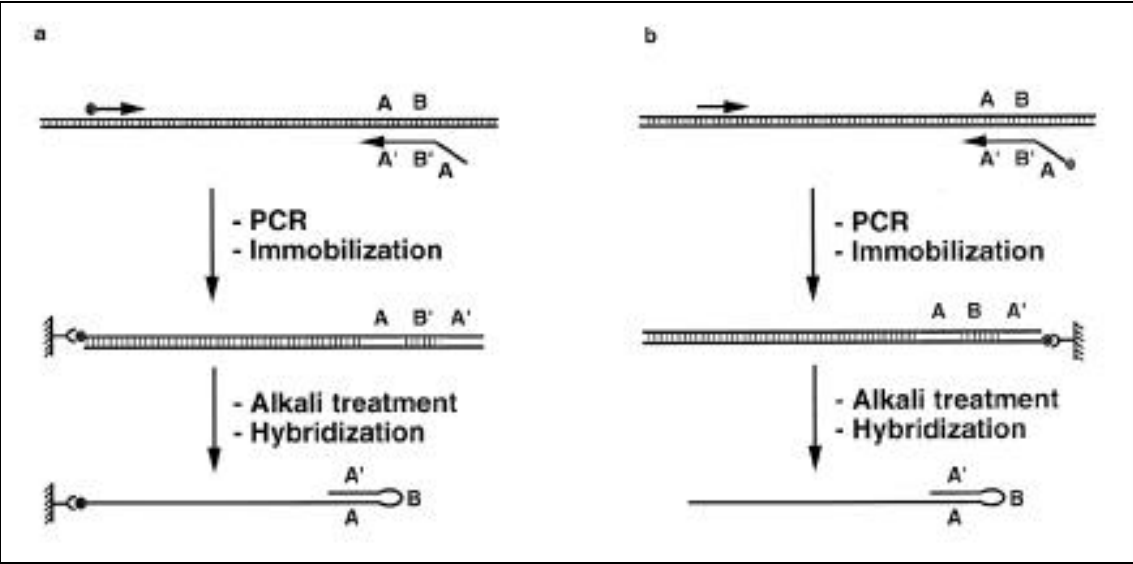

Figure 3. A schematic representation of stem-loop generation on genomic material by PCR. A primer containing a reverse complementary region at the $5^{\prime}$ end is used in the amplification. After amplification, the PCR product is immobilized on a solid-support, and a single-stranded template with a covalently attached primer was obtained after alkali treatment. Generation of (a) immobilized and (b) nonimmobilized loop-containing strand with free $3^{\prime}$ end that could be used for DNA sequencing is shown. $\mathrm{A}^{\prime}$ at the primer indicates template-specific sequence, $\mathrm{B}^{\prime}$ is the loop-forming region, and $\mathrm{A}$ and $\mathrm{B}$ are the reverse complementary parts of $\mathrm{A}^{\prime}$ and $\mathrm{B}^{\prime}$, respectively. The arrows indicate primer sites and the direction of synthesis. The blob indicates the biotin moiety. 
A signal in cycle 1 (Figure 5) is low, indicating as expected (2), that a portion of the PCR product contains an extra A added to the $3^{\prime}$ end by Taq DNA polymerase. A relatively high signalto-noise ratio is attained in liquid-phase pyrosequencing, although the signal intensity decreased in later cycles.

\section{DISCUSSION}

Here, two strategies for the generation of an intrinsic sequencing primer by PCR are described. The primer can be formed in the PCR by using either (i) a plasmid containing an inserted cassette downstream to the multi-cloning site that enables stem-loop structure formation or (ii) by introducing a region to the PCR arbitrary primer that is the reverse complement of the target DNA. After amplification and singlestrand elution, a region at the $3^{\prime}$ end of one of the strands forms a hairpin-like stem-loop structure with a free hydrox-

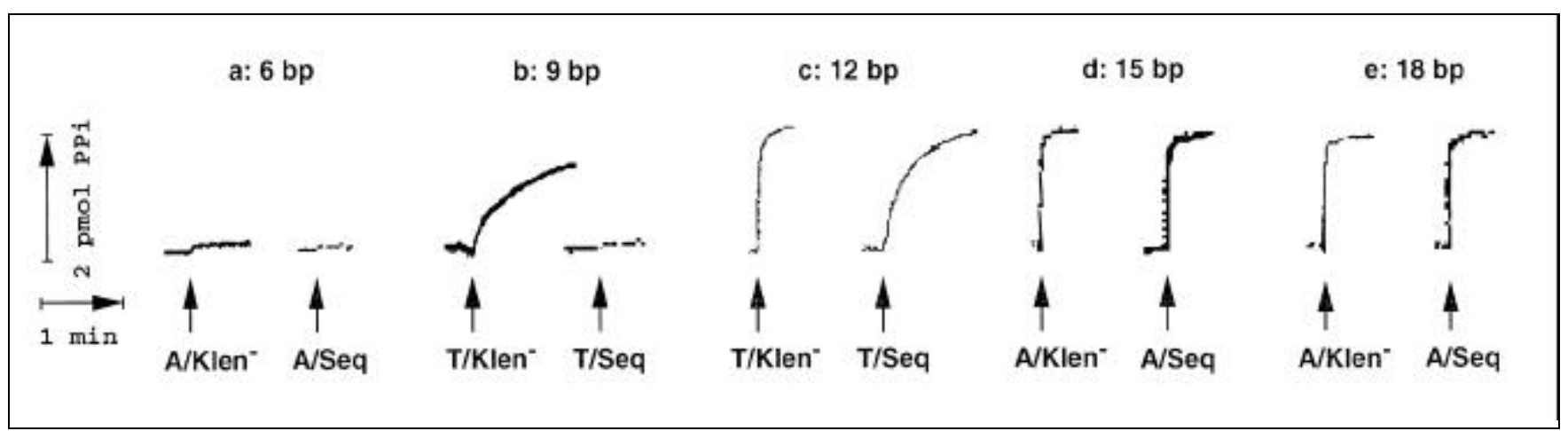

Figure 4. The effect of the stem-length on the polymerization efficiency. The polymerization rates on five different loop structures using Sequenase (Seq) and

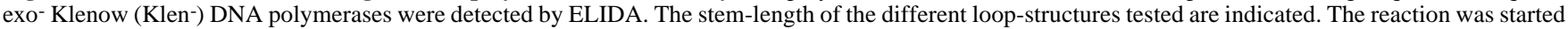
by the addition of $40 \mathrm{pmol}$ of the indicated deoxynucleotide. A and T indicate dATP and dTTP, respectively. 


\section{Research Reports}

yl group. With this approach, a primer that can serve as a start point for DNA polymerase is covalently bound to the template and cannot be detached from the template during the sequencing procedure. This is important when DNA sequencing is performed using sequencing-by-synthesis strategies. The detachment of the primer from the template will result in a decreased signal intensity. This problem is perhaps most pronounced when solid-phase pyrosequencing is performed and washing steps are needed after each extension step. Moreover, there is no need for any specific primer hybridization step because the covalently bound primer at the $3^{\prime}$ end is likely to hybridize spontaneously even at very low temperatures (Figure 2). Furthermore, the primer hybridizes with a high specificity, thus reducing the occurrence of false signals.

Among the parameters to be considered when constructing stem-loop structures for DNA sequencing are: $(i)$

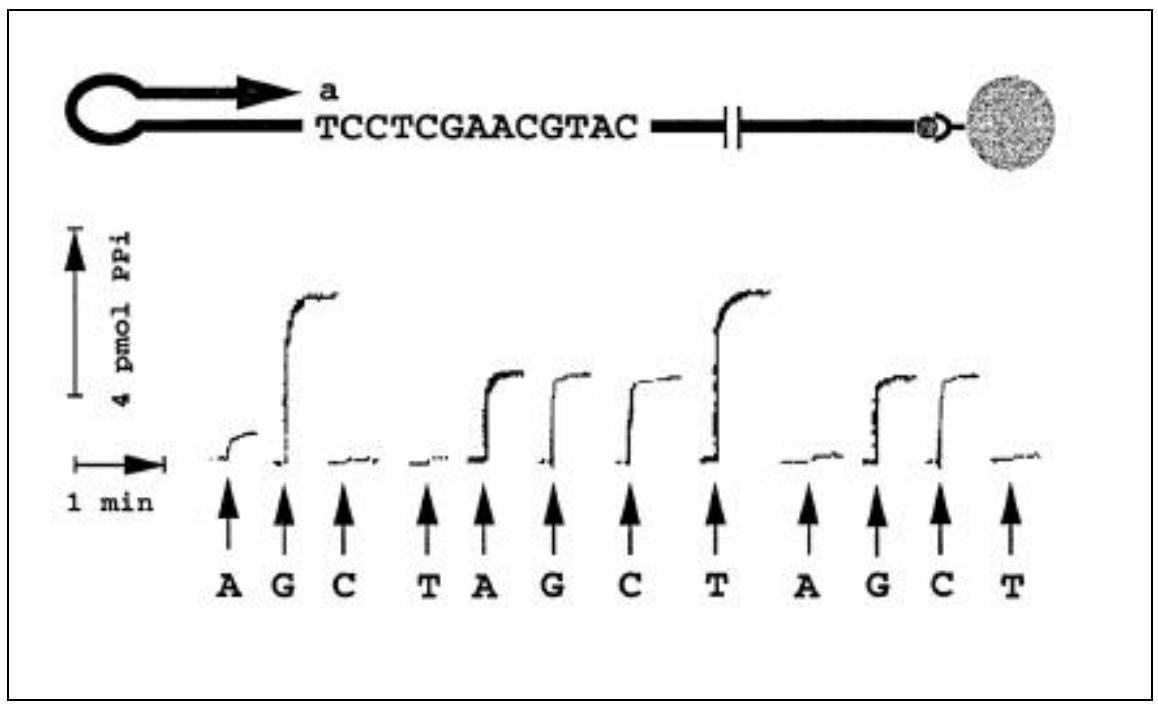

Figure 5. Solid-phase pyrosequencing performed on a PCR-generated loop structure. The PCR product was immobilized onto streptavidin-coated super paramagnetic beads. The single-stranded template making a stem of $15 \mathrm{bp}$ was incubated with exo- Klenow DNA polymerase. The reaction was started by addition of $40 \mathrm{pmol}$ of the indicated deoxynucleotide, and the PPi released was detected by ELIDA. The loop structure is designed to allow the addition of an extra A at the $3^{\prime}$ end during the PCR. The DNA sequence of the template is indicated. The first A signal is due to the lack of addition of an extra A on some of the templates during the PCR by the Taq DNA polymerase. The arrow indicates the direction of synthesis.

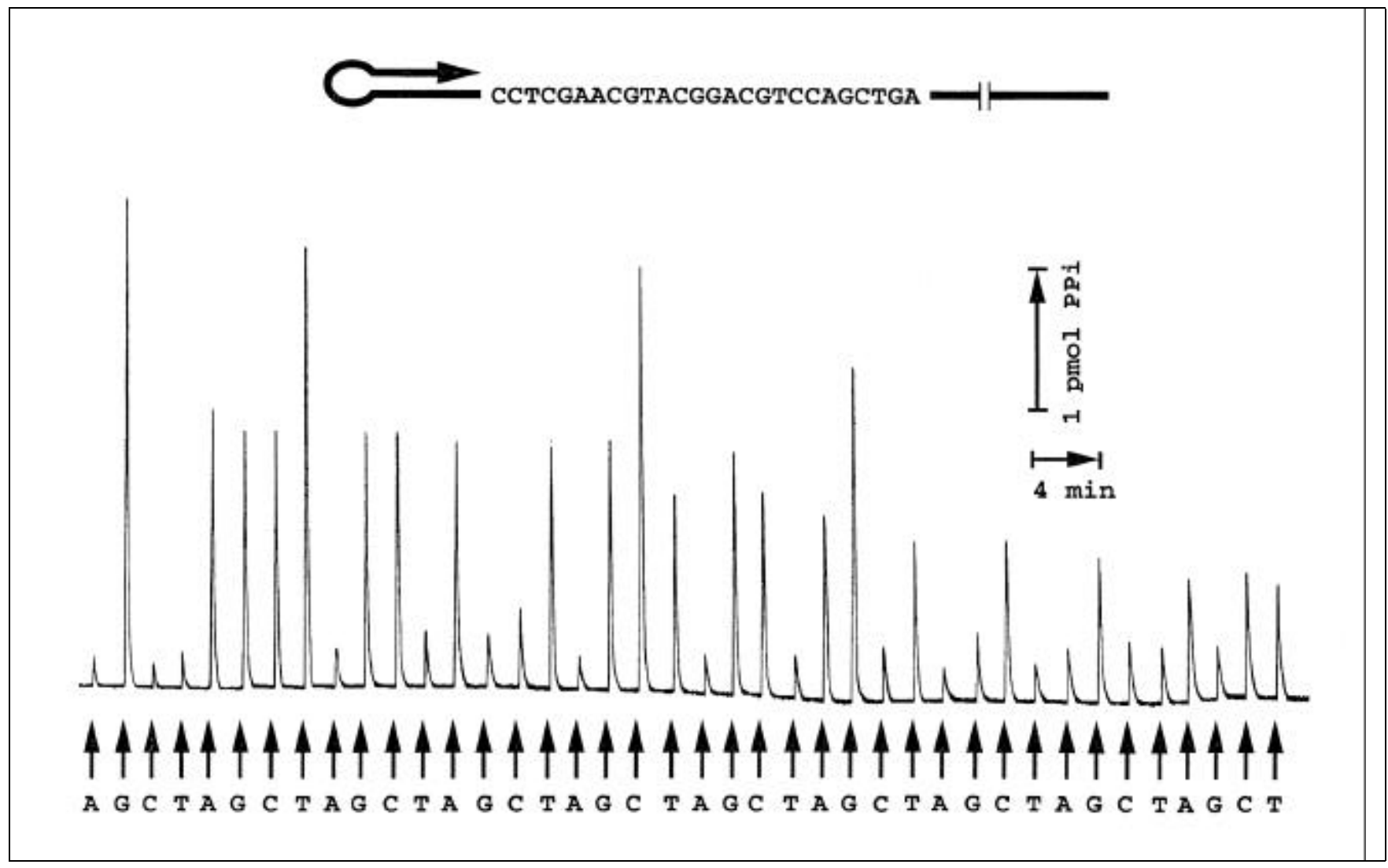

Figure 6. Liquid-phase pyrosequencing performed on a 161-base-long, self-primed PCR product. A PCR product (ca. 2 pmol), making a stem of 15 bp, was incubated with exo- Klenow DNA polymerase, luciferase, ATP sulfurylase and apyrase. The reaction was started by the addition of the indicated deoxynucleotide, and the PPi released was continuously detected as described earlier. The DNA sequence of the template is indicated. The arrow indicates the direction of synthesis. 
the nucleotide composition of the loop, (ii) the length of the stem after the loop and (iii) the choice of the polymerase and temperature profile in the PCR. The hairpin formation and stability is extremely dependent on both the central base sequence of the loop and the sequence of the stem region $(3,14)$. The effect of different nucleotide compositions on the loop stability have recently been studied $(15,16)$. Less stable loop structures with proper length of the stem might be preferable because a structure with a too stable stem can compete with the PCR-primer hybridization and the polymerase extension in the PCR step, thereby decreasing the efficiency of the PCR. It is important to use an exonuclease-proficient $\left(3^{\prime} \rightarrow 5^{\prime}\right)$ polymerase in the PCR, because most of the polymerases are showing terminal transferase activity that add an additional $\mathrm{A}$ at the 3 '-terminal end (2), and among those, the ex- onuclease-deficient polymerases are unable to remove the extra A. However, normal thermostable polymerases such as AmpliTaq can be used in the system if the PCR primer is designed to allow hybridization of the $3^{\prime}$ end of the primer to a region in which the first base of the template to be sequenced is a thymidine residue (Figure 5). Different polymerases require different stemlength for carrying out polymerization with a proper rate in the sequencing reaction. In this study, we found that Klenow DNA polymerase and Sequenase need a stem consisting of approximately 12 and 15 bases, respectively. This variation might depend on the difference in length of the template/primer involved in the protein/DNA interaction for different polymerases.

In conclusion, a method for the covalent incorporation of a sequencing primer has been developed. The primer can be introduced into both the immobilized and the non-immobilized DNA strand, thereby allowing bidirectional sequencing of both strands. The approach can be particularly useful for some sequencing methods such as pyrosequencing. The strategy facilitates automation of the template preparation procedure because the steps for primer addition and hybridization can be excluded.

\section{ACKNOWLEDGMENTS}

This work was supported by grants from Pyrosequencing AB, Axel och Margareta Ax:son Johnsons stiftelse för allmännyttiga ändamål and the Swedish Research Council for Engineering Sciences (TFR).

\section{REFERENCES}

1.Adams, M.D., J.M. Kelley, J.D. Gocayne, M. Dubnick, M.H. Polymeropoulos, H. Xiao, C.R. Merril, A. Wu, B. Olde and R.F. Moreno. 1991. Complementary DNA sequencing: expressed sequence tags and human genome project. Science 252:1651-1656.

2.Clark, J.M. 1988. Novel non-templated nucleotide addition reactions catalyzed by procaryotic and eucaryotic DNA polymerases. Nucleic Acids Res. 16:9677-9686.

3.Hald, M., J.B. Pedersen, P.C. Stein, F. Kirpekar and J.P. Jacobsen. 1995. A comparison of the hairpin stability of the palindromic d(CGCG(A/T)4CGCG) oligonucleotides. Nucleic Acids Res. 23:4576-4582.
4.Hengen, P.N. 1995. Vectorette, splinkerette and boomerang DNA amplification. Trends Biochem. Sci. 20:372-373.

5.Hultman, T., S. Bergh, T. Moks and M. Uhlén. 1991. Bidirectional solid-phase sequencing of in vitro-amplified plasmid DNA. BioTechniques 10:84-93.

6.Hultman, T., M. Murby, S. Stahl, E. Hornes and M. Uhlén. 1990. Solid phase in vitro mutagenesis using plasmid DNA template. $\mathrm{Nu}$ cleic Acids Res. 18:5107-5112.

7.Murray, V. 1989. Improved double-stranded DNA sequencing using the linear polymerase chain reaction. Nucleic Acids Res. 17:8889.

8.Nyrén, P. and A. Lundin. 1985. Enzymatic method for continuous monitoring of inorganic pyrophosphate synthesis. Anal. Biochem. 151:504-509.

9.Ronaghi, M., S. Karamohamed, B. Pettersson, M. Uhlén and P. Nyrén. 1996. Real-time DNA sequencing using detection of pyrophosphate release. Anal. Biochem. 242:84-89.

10.Ronaghi, M., M. Uhlén and P. Nyrén. 1998. Pyrosequencing, a novel DNA sequencing method based on pyrophosphate detection. Science 281:363-365.

11.Sambrook, J., E.F. Fritsch and T. Maniatis. 1989. Molecular Cloning: A Laboratory Manual. 2nd ed., CSH Laboratory Press, Cold Spring Harbor, NY

12.Sanger, F., S. Nicklen and A.R. Coulson. 1977. DNA sequencing with chain-terminating inhibitors. Proc. Natl. Acad. Sci. USA 74:5463-5467.

13.Smith, L.M., S. Fung, M.W. Hunkapiller, T.J. Hunkapiller and L.E. Hood. 1985. The synthesis of oligonucleotides containing an aliphatic amino group at the $5^{\prime}$ terminus: synthesis of fluorescent DNA primers for use in DNA sequence analysis. Nucleic Acids Res. 13:2399-2412.

14. Tanikawa, J., Y. Nishimura, I. Hirao and K. Miura. 1991. NMR spectroscopic study of single-stranded DNA fragments of d(CGGCGAAAGCCG) and d(CGGCAAAAGCCG). Nucleic Acids Symp. Ser. 25:47-48.

15. Yamakawa, H., D. Nakajima and O. Ohara. 1996. Identification of sequence motifs causing band compressions on human cDNA sequencing. DNA Res. 3:81-86.

16.Yoshizawa, S., G. Kawai, K. Watanabe, K. Miura and I. Hirao. 1997. GNA trinucleotide loop sequences producing extraordinarily stable DNA minihairpins. Biochemistry 36:4761-4767.

17.Zimmermann, J., H. Voss, C. Schwager, J. Stegemann and W. Ansorge. 1988. Automated Sanger dideoxy sequencing reaction protocol. FEBS Lett. 233:432-436.

Received 1 May 1998; accepted 16 July 1998.

\section{Address correspondence to:}

Dr. Pål Nyrén

Department of Biotechnology

The Royal Institute of Technology

SE-100 44 Stockholm, Sweden

Internet:paaln@biochem.kth.se 